\title{
Metabolic adjustment enhances food web stability
}

\author{
Pierre Quévreux ${ }^{1}$ and Ulrich Brose ${ }^{2,3}$
}

${ }^{1}$ Sorbonne Universités, Sorbonne Paris Cité, Paris Diderot Univ Paris 07,

CNRS, INRA, IRD, UPEC, Institut d'Écologie et des Sciences de

l'Environnement - Paris, iEES-Paris, 4 place Jussieu, F-75252 Paris,

\section{France}

${ }^{2}$ German Centre for Integrative Biodiversity Research (iDiv)

Halle-Jena-Leipzig, Deutscher Platz 5e, 04103, Leipzig, Germany

${ }^{3}$ Institute of Ecology, Friedrich Schiller University Jena, Dornburger-Str.

159, 07743, Jena, Germany

pierre.quevreux@cri-paris.org

orcid.org/0000-0002-3531-1410

ulrich.brose@idiv.de

orcid.org/0000-0001-9156-583X 
bioRxiv preprint doi: https://doi.org/10.1101/276550; this version posted March 5, 2018. The copyright holder for this preprint (which was not certified by peer review) is the author/funder, who has granted bioRxiv a license to display the preprint in perpetuity. It is made available under aCC-BY 4.0 International license.

\section{Abstract}

2 Understanding ecosystem stability is one of the greatest challenges of ecology. Over several

3 decades, it has been shown that allometric scaling of biological rates and feeding inter-

4 actions provide stability to complex food web models. Moreover, introducing adaptive

${ }_{5}$ responses of organisms to environmental changes (e.g. like adaptive foraging that enables

6 organisms to adapt their diets depending on resources abundance) improved species per-

7 sistence in food webs. Here, we introduce the concept of metabolic adjustment, i.e. the

8 ability of species to slow down their metabolic rates when facing starvation and to increase

9 it in time of plenty. We study the reactions of such a model to nutrient enrichment and the adjustment speed of metabolic rates. We found that increasing nutrient enrichment

11 leads to a paradox of enrichment (increase in biomasses and oscillation amplitudes and ultimately extinction of species) but metabolic adjustment stabilises the system by dampening the oscillations. Metabolic adjustment also increases the average biomass of the top

14 predator in a tri-trophic food chain. In complex food webs, metabolic adjustment has a stabilising effect as it promotes species survival by creating a large diversity of metabolic rates. However, this stabilising effect is mitigated in enriched ecosystems. Phenotypic plasticity of organisms must be considered in food web models to better understand the response of organisms to their environment. As metabolic rate is central in describing biological rates, we must pay attention to its variations to fully understand the population dynamics of natural communities. 
bioRxiv preprint doi: https://doi.org/10.1101/276550; this version posted March 5, 2018. The copyright holder for this preprint (which was

not certified by peer review) is the author/funder, who has granted bioRxiv a license to display the preprint in perpetuity. It is made available under aCC-BY 4.0 International license.

\section{${ }_{21}$ Introduction}

42

Identifying the mechanisms responsible for ecosystem stability is one of the main scientific tasks in ecology (de Ruiter, 2005; Montoya et al., 2006; Rooney and McCann, 2012; Loreau and de Mazancourt, 2013). Natural ecosystems are assumed to be stable (in the sense of dynamic stability, defined by the equilibrium stability and the variability (Pimm, 1984; McCann, 2000)) thanks to many mechanisms resulting from the diversity of interacting species (MacArthur, 1955; Elton, 1958). However, mathematical models of ecosystems predicted opposite results. For instance, the theoretical study performed by May (1972) demonstrated that diversity, complexity (measured by the linkage probability between pairs of species) and the average interaction strength decreased the stability of random interaction networks (assessed by a linear stability analysis). Subsequently, many mechanisms promoting food web stability were identified and two of them inspired us to implement a new one in food web models. The first mechanism is the allometric scaling of biological rates (e.g. metabolic rate, feeding strength), describing them as power functions of individual body mass (Yodzis and Innes, 1992; Brown et al., 2004; Savage et al., 2004; Brose et al., 2008; Pawar et al., 2012; Kalinkat et al., 2013). These relationships provided a better prediction of species biomasses in empirical data than any other model parametrisation (Boit et al., 2012; Hudson and Reuman, 2013). In addition, allometric scaling coupled with size structured communities (i.e. consumers larger than their prey) 40 lead to more stable food webs with fewer extinctions (Brose et al., 2006; Brose, 2008; $41 \quad$ Kartascheff et al., 2010).

${ }_{3}$ The second mechanism is adaptive foraging. Kondoh (2003) included adaptive forag44 ing behaviour into food web models to enable the consumers to maximise their biomass 45 income by preferentially hunting more abundant prey. The result is dramatic, with a 
bioRxiv preprint doi: https://doi.org/10.1101/276550; this version posted March 5,2018 . The copyright holder for this preprint (which was not certified by peer review) is the author/funder, who has granted bioRxiv a license to display the preprint in perpetuity. It is made available under aCC-BY 4.0 International license.

reversion of the pattern predicted by May: with adaptive foragers, increasing species richness and complexity enhances species persistence. Furthermore, food webs with randomly set interactions and adaptive foraging converge towards size-structured food webs with predators systematically larger than their prey (Heckmann et al., 2012). In such models, species biomasses are not the only dynamic variables, food web structures and interaction parameters are also dynamic (de Ruiter, 2005). However, one central parameter has always been considered constant in food web models: the metabolic rate. The closest examples to adjustable metabolic rates were given by Kuwamura et al. (2009), Nakazawa et al. (2011) and Wang and Jiang (2014) who considered simple models with a structured population of Daphnia including metabolically active adults and dormant eggs. In nature, however, many organisms exhibit phenotypic plasticity in the expression of metabolism (Brown et al., 2004; Glazier, 2005; Jeyasingh, 2007; Glazier, 2009a; Carey et al., 2013; Norin et al., 2015). In fact, Makarieva et al. (2005) pointed out that organisms with different body sizes can display similar metabolic rates depending on their activity. Moreover, animals from all major phyla are able to slow down their activity to face harsh conditions such as drought and starvation using body mass reduction (DeLong et al., 2014b), torpor, diapause (depression of 60-95\%) or cryptobiosis (depression of 99100\%) (Guppy and Withers, 1999). Considering the metabolic activity of organisms as a constant parameter is a strong assumption despite its central role in food web models. In this study, we model the plastic response of metabolism similarly to adaptive foraging. As adaptive foraging maximises the growth rate of consumers by varying the foraging effort 67 for the different prey, we propose that metabolic adjustment maximises the growth rate 68 by varying the metabolic rate. 
bioRxiv preprint doi: https://doi.org/10.1101/276550; this version posted March 5, 2018. The copyright holder for this preprint (which was not certified by peer review) is the author/funder, who has granted bioRxiv a license to display the preprint in perpetuity. It is made available under aCC-BY 4.0 International license.

71 justable metabolism for food web models. First, this adjustable behaviour should have a

72 of the prey increases, consumers will raise their metabolic activity that is directly related to their consumption rate. The consequence is an increase in the predation pressure and top-down control imposed by consumers on their prey at high densities. On the contrary, in periods of starvation, consumers slow down their metabolic rate to minimise their loss in biomass caused by respiration, which keeps predator biomasses at a level high enough to avoid extinction (Chesson and Huntly, 1989; Polis et al., 1996; Chesson, 2000). In this study, we wonder whether the combination of these two effects stabilises the dynamics of the species (decreased amplitude and increased minima of population oscillations). In consequence our second prediction is that adaptive metabolic rates increase the persistence of complex food webs. As a measure of stability, we use the time variability of species biomasses (existence of fixed points and amplitude of biomass oscillation) and species persistence (proportion of surviving species in a food web).

\section{Material and Methods}

We study the impact of metabolic adjustment on a simple tri-trophic food chain and complex food webs. Both are modified versions of the Allometric Trophic Network (ATN) (Brose et al., 2006). The complex food webs rely on the Williams and Martinez (2000) niche model for their structure and on the Yodzis and Innes (1992) predator-prey model for the dynamic equations and their parameters.

\section{Food web construction}

92 The construction of the complex food webs follows the niche model (Williams and Martinez, 2000; Brose et al., 2006; Heckmann et al., 2012; Binzer et al., 2016) as it successfully 
predicted the food web structures of natural communities. The trophic interactions across species are set according to the algorithm detailed by Williams and Martinez (2000) with an expected connectance equal to 0.15 . The basal species described by Williams and Martinez (2000) are set as primary producers and the others as consumers. The niche values $n_{i}$ (uniformly drawn in a $[0,1]$ interval for each of the 40 initial species) used to parametrise the niche model are also used to calculate species body mass as follows (Heckmann et al., 2012).

$$
M_{i}=10^{N \cdot n_{i}}
$$

Here $N$ is equal to 6 , that means the biggest species is one million times larger than the smallest ones.

\section{Predator-prey model}

The population dynamics of the food web follows the ATN model (Brose et al., 2006; Williams et al., 2007).

$$
\begin{aligned}
\frac{d B_{i}}{d t} & =r_{i} G_{i} B_{i}-\sum_{j=\text { consumers }} x_{j} y_{j} B_{j} F_{j i} / e_{j i} \\
\frac{d B_{i}}{d t} & =-x_{i} B_{i}+\sum_{j=\text { prey }} x_{i} y_{i} B_{i} F_{i j}-\sum_{j=\text { consumers }} x_{j} y_{j} B_{j} F_{j i} / e_{j i}
\end{aligned}
$$

These equations describe changes in relative, biomass densities of primary producers (2a) and consumer species $(2 \mathrm{~b})$. In these equations $B_{i}$ is the biomass of species $i, r_{i}$ is the mass-specific maximum growth rate of primary producers, $G_{i}$ is the logistic growth rate of primary producers (Equation (3)), $x_{i}$ is $i$ 's mass-specific metabolic rate, $y_{i}$ is the maxi- 
mum consumption rate of consumers relative to their metabolic rate, $e_{j i}$ is $j$ 's assimilation efficiency when consuming population $i$ and $F_{i j}$ describes the realised fraction of $i$ 's maximum rate of consumption achieved when consuming $j$ (equation (4)). Primary producers growth rate is modelled by a logistic growth with a shared carrying capacity $K$ which ensures a comparable primary production among food webs, regardless the number of primary producers (equation 3).

$$
G_{i}=\left(K-\sum_{\substack{j=\text { primary } \\ \text { producers }}} B_{j}\right) / K
$$

The consumption rate of prey depends on a Holling type II functional response with predator interference (Equation (4)). The preference of consumers for their prey $\omega_{i j}$ are set to $1 / p_{i}$ with $p_{i}$ the number of consumer $i$ 's prey as we have no a priori information on preferences. Thus, all consumption rates are only driven by consumer body masses and prey biomass densities. $\omega_{i j}$ are recalculated after each extinction to follow the changes of the number of prey $p_{i}$.

$$
F_{i j}=\frac{\omega_{i j} B_{j}}{B_{0}+c B_{i} B_{0}+\sum_{k=\text { prey }} \omega_{i k} B_{k}}
$$

Here $B_{0}$ is the half-saturation density of $i$ and $c$ the predator interference.

Basically, mass specific biological rates (biomass production, metabolic rate and maximum consumption rate) follow the negative-quarter power-law relationship with species body masses as described by the metabolic theory of ecology (Brown et al., 2004; Savage et al., 2004). The time scale of the system is defined by normalising the biological rates to the mass-specific growth rate of the smallest primary producer as performed by Yodzis and 
Innes (1992); Brose et al. (2006); Williams et al. (2007) (Equations 5a and 5b). Then the maximum consumption rates are normalised by the metabolic rates (Equations 5c). Thus, the loss due to respiration and the gain due to consumption both directly depend on the metabolic rate (Equation $(2 b)$ ).

$$
\begin{aligned}
r_{i} & =\left(\frac{M_{i}}{M_{r e f}}\right)^{-0.25} \\
x_{i} & =\frac{a_{x}}{a_{r}}\left(\frac{M_{i}}{M_{r e f}}\right)^{-0.25} \\
y_{i} & =\frac{a_{y}}{a_{x}}
\end{aligned}
$$

With $M$ the body mass of species $i, M_{r e f}$ the body mass of the smallest primary producer, $a_{r}, a_{x}$ and $a_{y}$ are allometric constants (see Brose et al. (2006) and Williams et al. (2007) for more details on the normalisation).

\section{Metabolic adjustment model}

We propose to model the metabolic adjustment by an optimisation of the mass-specific net growth rate $g_{i}$ as in adaptive foraging models (Kondoh, 2003; Uchida et al., 2007) or in body mass plasticity models (DeLong et al., 2014b). Thus, the consumer adjusts its metabolic rate to maximise the balance between ingestion and respiration that both depend on metabolic rate. Metabolic adjustment does not apply to primary producers that are considered as basal resources species with constant resource supply (Equation 


$$
\begin{aligned}
\frac{d x_{i}}{d t} & =x_{i} X \frac{\partial g_{i}}{\partial x_{i}}=x_{i} X\left(-1+\sum_{j=p r e y} e_{i j} y F_{i j}\right) \\
g_{i} & =-x_{i}+\sum_{j=\text { prey }} e_{i j} x_{i} y F_{i j}
\end{aligned}
$$

With $X$ the metabolic adjustment coefficient representing the speed of the adjustment. The higher $X$ is, the faster the response of species to modifications of their growth rate is. The metabolic rate is bounded by 1 and 0.001 to ensure a minimum metabolic rate and to prevent a destabilising high metabolic rate. The values predicted by the equation $5 \mathrm{~b}$ fall in this interval that is consistent with Makarieva et al. (2005) (Supplementary material Appendix B, Fig.B5, B6,B7,B8).

\section{Simulations}

The model is coded in $C++$ and the simulations performed with the $G S L$ ODE solver. The simple tri-trophic food chain only contains a primary producer, a herbivore and a carnivore. Their body masses are respectively set to $1,10^{2}$ and $10^{4}$. For the complex food webs, each simulation is independent from the other and only differs in the body mass distribution and the architecture of the food web. The system starts with 40 species with initial biomass of 0.1 and the metabolic rates are initialised with the values predicted by the metabolic theory of ecology (Equation 5). The simulations are performed for 10,000 time steps and only the last 1000 steps are recorded. Species persistence is the proportion of the 40 initial species that survives until the end of the simulation. Each combination of parameters is tested for 100 different food webs. 


\section{Results}

\section{Effect of adaptive metabolic rate on species dynamics}

The first system we consider is a simple tri-trophic food chain containing a primary producer, a herbivore and a carnivore. The effects of the resource availability on species dynamics are represented by bifurcation diagrams (Fig.1). The food chain without metabolic adjustment $(X=0)$ displays large biomass oscillations whose amplitude increases with the carrying capacity $K$ (Fig.1A) and the minima reaches extremely low values, especially for the herbivore (Supplementary material Appendix A, Fig.A1A). As there is no metabolic adjustment, the metabolic rates are constant (Fig.1B) and their values are those predicted by the metabolic theory of ecology (Equations 5a,b,c). The food chain with metabolic adjustment $(X=2)$ has fixed points for $K \leq 7$ and oscillations for $K>7$ (Fig. 1A). Despite the multi-period oscillations, the system is not chaotic (Supplementary material Appendix A, Fig.A4A). The amplitude of oscillations increases with the carrying capacity for all species but remains lower than in the food chain without metabolic adjustment. The biomass minima increases with higher values of the metabolic adjustment coefficient (Supplementary material Appendix A, Fig.A1A). The herbivore metabolic rate remains constantly at the maximum value allowed by the model, whereas the carnivore metabolic rate increases with carrying capacity $K$ until it oscillates for $K>7$ (Fig.1B).

The tri-trophic food chain has fixed points along a gradient in metabolic adjustment coefficients for a carrying capacity $K=2$ (Fig.2), except for $X=0$ (origin of the x-axis corresponding to the situation described in Fig.1A). Increasing the metabolic adjustment coefficient increases the biomass of the herbivore and of the carnivore while it decreases the biomass of the primary producer. However, we observe an increase in the primary producer biomass and a decrease in the herbivore biomass for the low values of $X$. The 
metabolic rate of the herbivore is maximum for $X>0$ and the metabolic rate of the carnivore first sharply increases with the increasing metabolic adjustment coefficient $X$ and then it decreases (Fig.2B). The response is similar for $K=5$ and $X<4$ but for $X \geq 4$ the system oscillates (Fig.2A), yet it is not chaotic (Supplementary material Appendix A, Fig.A4B). Increasing the metabolic adjustment coefficient does not increase the amplitude of biomass oscillations, it even decreases them for the primary producer. The biomass of the carnivore increases with $X$, the amplitude of the oscillations of its metabolic rate increases (Fig.2B) while the amplitude of its biomass oscillations remains mostly unchanged. Increasing the metabolic adjustment coefficient also increases the biomass minima of each species (Supplementary material Appendix A, Fig.A1B).

\section{Effect of adaptive metabolic rates on persistence}

The response of stability to metabolic adjustment and enrichment in complex food webs is assessed through the average species persistence (Fig.3A). In food webs without metabolic adjustment $(X=0)$, increasing $K$ does not significantly change species persistence that stays around 0.3 . In food webs with metabolic adjustment $(X>0)$, for a fixed carrying capacity $K$, increasing $X$ promotes species persistence, especially at low values of $K$ where all species can survive. If $K>3$, species persistence first decreases and then increases as $X$ increases. For a fixed value of $X$, increasing $K$ decreases species persistence and thus leads to an example of the paradox of enrichment. To sum up, enrichment through the increase of the carrying capacity has a destabilising effect on species persistence, whereas metabolic adjustment increases it substantially. 
biomass $\left(<10^{-2}\right)$ and a low metabolic rate $\left(<10^{-2.5}\right)$ and 'fast species' with a high biomass $\left(>10^{-2}\right)$ and a high metabolic rate $\left(>10^{-2.5}\right)$ (Fig.3B and 3C). Increasing the carrying capacity $K$ does not seem to change the repartition of species in these two categories (Fig.3B) while more species are in an intermediate category (low biomass and high metabolic rate) at low values of metabolic adjustment coefficient $X$ (Fig.3C). This difference is confirmed in Fig.3D where three groups of species can be identified for $X>0.002$ : (a) species with minimum or low metabolic rate, (b) species with intermediate metabolic rate and (c) species with maximum metabolic rate. (a) species correspond to the slow species, (b) and (c) to the fast species. Such a non-differentiation of the metabolic profile of species for low metabolic adjustment coefficients may be the origin of the first decrease of species persistence with increasing $X$ for $K>3$ (Fig.3A).

\section{Discussion}

We studied the consequences of an adaptive metabolic rate for different aspects of food web stability. We predicted that metabolic adjustment enables species to fit their metabolic rate to their energy budget and the resource availability. In times of bonanza, it allows species to increase their activity and then to exploit more resources. In harsh times, however, metabolic adjustment also lets organisms slow down their activity to save their energy until the next season of plenty (Polis et al., 1996). This behaviour is typically the case for microbial organisms that can get encysted or can produce spores (Dawes and Ribbons, 1962; Fenchel and Finlay, 1983; Glazier, 2009b) but also larger organisms that can shift between resting and activity metabolism (Glazier, 2008; Hudson et al., 2013) or hibernating (Guppy and Withers, 1999). In the case of our models, adjustable metabolic rates reduce the magnitude of biomass oscillations and increase the average biomass of carnivores. Additionally, they greatly increase the stability of complex food webs by 
increasing species persistence at low resource densities.

\section{Effect of adaptive metabolic rate on species dynamics}

Our first aim was to provide a mechanistic insight in the consequences of metabolic adjustment for population dynamics. We followed prior studies employing tri-trophic food chains with allometric scaling of population parameters, which provides a fully deterministic and easily tractable system (Otto et al., 2007; Binzer et al., 2012). First, enrichment, through the increase of the carrying capacity $K$, has a destabilising effect on population dynamics (Rall et al., 2008; Schwarzmüller et al., 2015). Such a destabilisation, called paradox of enrichment, is due to the unbalance between the growth and the mortality of organisms (Rosenzweig, 1971; DeAngelis, 1992; Roy and Chattopadhyay, 2007; Rip and McCann, 2011). However, this destabilising effect is dampened by metabolic adjustment that promotes fixed points or reduces the amplitude of biomass oscillations and increases the biomass minima. Increasing the speed of adjustment (i.e. increasing the metabolic adjustment coefficient $X$ ) is destabilising because it promotes biomass oscillations, but it also increases the biomass of carnivores. We can compare our results to prior studies using adaptive foraging that inspired our modelling of metabolic adjustment (Kondoh, 2003, 2010; Křivan and Diehl, 2005; Mougi and Nishimura, 2008). The adaptability of predator attack rates or prey defences (Vos et al., 2004; Verschoor et al., 2004) also decreases in the amplitude of biomass oscillations, increases the average biomass of carnivores and keeps the minima away from the extinction threshold (Mougi and Nishimura, 2007). The outcome of these processes are similar because both rely on growth rate optimisation, which seems to highly improve the persistence of higher trophic levels that are generally most prone to extinction (Binzer et al., 2011). However, metabolic adjustment affects both the growth and the mortality rates of consumers while adaptive foraging only 
increases the growth rate and inducible defences decrease the mortality rate. In consequence, adaptive metabolic rates enables a better control of species dynamics, especially

for top consumers whose loss rate only depends on metabolic rate and not on predation. In our tri-trophic food chain, carnivores have a highly variable metabolic rate while the herbivore's metabolic rate always stays at the upper limit of metabolic rate range. This can be attributed to a trophic cascade: the carnivore controls the herbivore population and the primary producer thrives. Thus, the herbivore always has plenty of resources, and increasing the metabolic rate increases more the ingestion rate and the growth rate compared to the loss rate.

\section{Effect of adaptive metabolic rate on species persistence}

Our second aim was to address the impact of an adjustable metabolic rate on the species persistence of complex food webs. The null model is a classic allometric model (Brose et al., 2006) that displays an increase in persistence with increasing carrying capacity and increase in the energy flow in the system (Dunne et al., 2005; Rall et al., 2008). As expected, adding an adjustable metabolic rate increases the species persistence at low resources levels. Similarly to the results of studies on adaptive foraging (Kondoh, 2003; Heckmann et al., 2012), higher adjustment coefficients (the metabolic adjustment in our case) increase species persistence. Such an increase in persistence can be partially attributed to the slow species with a low biomass and a low metabolic rate described in our study. However, no positive relationship between density and metabolic rate has been reported in previous studies (DeLong et al., 2014a; Yashchenko et al., 2016). Alternatively, these slow species could just be slow in getting extinct because of their very low metabolic rate (which is the loss rate in our model). However, the large diversity of metabolic rates in the fast species enables these species to better adapt to the specific situation concerning 
top-down control and resource availability of each food web, leading to an increased species persistence. The improvement in species persistence by the metabolic adjustment slips away as the carrying capacity increases. Our results obtained for the tri-trophic food chain demonstrate that metabolic adjustment dampens the paradox of enrichment but does not resolve it as in models with adaptive foraging (Mougi and Nishimura, 2007, 2008).

\section{Conclusion and perspectives}

Previous models studied mechanisms similar to the metabolic adjustment by using structured populations of consumers with active adults and dormant eggs (Kuwamura et al., 2009; Nakazawa et al., 2011; Wang and Jiang, 2014). In these models, the resting eggs act as a refuge for the consumer, enabling them to escape from starvation. This mechanisms is very different of our representation of metabolic adjustment because metabolic adjustment is an energy budget optimisation process while the production of resting eggs forms a seed bank maintaining a high biodiversity (Jones and Lennon, 2010). This difference is emphasised by our divergent results. In fact, Nakazawa et al. (2011) found that the production of resting eggs leads to more stable population dynamics as it responds more to seasonality than to non-seasonal variation in resource availability (in this case the effect of resting eggs is weak). Metabolic adjustment (i.e. response to resource availability) in food webs deeply changes the outcome of the model. In fact, adjustable metabolic rates greatly increase stability regarding many criteria: they increase the average biomass of top trophic levels, decrease the variability in population biomass density and increase the minima of population biomass density, keeping them away from the extinction threshold. Including metabolic adjustment in food web models improves the representation of the diversity of organisms whose metabolic activity is not predicted by the metabolic theory of ecology (Guppy and Withers, 1999; Glazier, 2005; Makarieva et al., 2008; DeLong et al., 
2014b). More broadly, considering phenotypic plasticity (as it was extensively done for adaptive foraging or inducible defences for instance) is crucial to better understand the fast response of organisms to environmental changes (Marshall and McQuaid, 2011; Marshall et al., 2011; Magozzi and Calosi, 2015). Interesting future directions in this research agenda would be to extend metabolic adjustment to primary producers depending on the supply of non-biotic resources affected by seasonality (e.g. nutrients, sun light, water...) or to include more parameters such as the attack rate in the list of biological rates directly affected by the adjustable metabolic rate. Finally, it would also be interesting to set the metabolic adjustment coefficient $X$ as an allometric parameter because single cell organisms are expected to respond faster than large animals for instance. Overall, adjustable metabolic rates holds great potential to represent the biology of many species in natural communities as metabolic rate plays a central role in describing species biological functions.

\section{Acknowledgments}

We would like to thank Christoph Digel and Björn Rall for their help during this study. I would also like to thank members of the journal club of the iEES and Marie-Hélène Berthet for their helpful review. We thank the École Normale Supérieure and the PhD program "Ecole Doctorale Frontières du Vivant (FdV) - Programme Bettencourt" for their financial support. U.B. acknowledges support by the German Research Foundation (FZT 118).

\section{Data accessibility}

All data are included in the manuscript and its supporting information. The codes are available on Zenodo and GitHub (doi:10.5281/zenodo.1170138). 


\section{Supplementary materials}

Supplementary material (available online as Appendix XXXXX (insert manuscript number) at LÄNK). Appendix 1-2

\section{References}

Benettin, G., Galgani, L., Giorgilli, A. and Strelcyn, J.-M. 1980. Lyapunov Characteristic Exponents for smooth dynamical systems and for hamiltonian systems; a method for computing all of them. Part 1: Theory. - Meccanica 15(1): 9-20.

Binzer, A., Brose, U., Curtsdotter, A., Eklöf, A., Rall, B. C., Riede, J. O. and de Castro, F. 2011. The susceptibility of species to extinctions in model communities. - Basic and Applied Ecology 12(7): 590-599.

Binzer, A., Guill, C., Brose, U. and Rall, B. C. 2012. The dynamics of food chains under climate change and nutrient enrichment. - Philosophical Transactions of the Royal Society B: Biological Sciences 367(1605): 2935-2944.

Binzer, A., Guill, C., Rall, B. C. and Brose, U. 2016. Interactive effects of warming, eutrophication and size structure: impacts on biodiversity and food-web structure. Global Change Biology 22(1): 220-227.

Boit, A., Martinez, N. D., Williams, R. J. and Gaedke, U. 2012. Mechanistic theory and modelling of complex food-web dynamics in Lake Constance. - Ecology Letters 15(6): 594-602.

Brose, U. 2008. Complex food webs prevent competitive exclusion among producer species. - Proceedings of the Royal Society of London B: Biological Sciences 275(1650): $2507-2514$. 
Brose, U., Ehnes, R. B., Rall, B. C., Vucic-Pestic, O., Berlow, E. L. and Scheu, S. 2008. Foraging theory predicts predator-prey energy fluxes. - Journal of Animal Ecology 77(5): 1072-1078.

Brose, U., Williams, R. J. and Martinez, N. D. 2006. Allometric scaling enhances stability in complex food webs. - Ecology Letters 9(11): 1228-1236.

Brown, J. H., Gillooly, J. F., Allen, A. P., Savage, V. M. and West, G. B. 2004. Toward a metabolic theory of ecology. - Ecology 85(7): 1771-1789.

Carey, N., Sigwart, J. D. and Richards, J. G. 2013. Economies of scaling: More evidence that allometry of metabolism is linked to activity, metabolic rate and habitat. - Journal of Experimental Marine Biology and Ecology 439: 7-14.

Chesson, P. 2000. Mechanisms of maintenance of species diversity. - Annual Review of Ecology and Systematics 31(1): 343-366.

Chesson, P. and Huntly, N. 1989. Short-term instabilities and long-term community dynamics. - Trends in Ecology \& Evolution 4(10): 293-298.

Dawes, E. A. and Ribbons, D. W. 1962. The endogenous metabolism of microorganisms. Annual Review of Microbiology 16(1): 241-264.

DeAngelis, D. L. 1992. Dynamics of nutrient cycling and food webs. - No. 9 in Population and community biology series. Chapman \& Hall, 1st ed.

DeLong, J. P., Hanley, T. C. and Vasseur, D. A. 2014a. Competition and the density dependence of metabolic rates. - Journal of Animal Ecology 83(1): 51-58.

— 2014b. Predator-prey dynamics and the plasticity of predator body size. - Functional Ecology 28(2): 487-493. 
Dunne, J. A., Brose, U., Williams, R. J. and Martinez, N. D. 2005. Modeling food web dynamics: complexity-stability implications. - In: Aquatic Food Webs. Oxford University Press, pp. 117-129.

Elton, C. S. 1958. The ecology of invasions by animals and plants. - Chapman \& Hall.

Fenchel, T. and Finlay, B. J. 1983. Respiration rates in heterotrophic, free-living protozoa. - Microbial Ecology 9(2): 99-122.

Glazier, D. S. 2005. Beyond the : variation in the intra- and interspecific scaling of metabolic rate in animals. - Biological Reviews 80(04): 611.

— 2008. Effects of metabolic level on the body size scaling of metabolic rate in birds and mammals. - Proceedings of the Royal Society B: Biological Sciences 275(1641): 1405-1410.

— 2009a. Activity affects intraspecific body-size scaling of metabolic rate in ectothermic animals. - Journal of Comparative Physiology B 179(7): 821-828.

— 2009b. Metabolic level and size scaling of rates of respiration and growth in unicellular organisms. - Functional Ecology 23(5): 963-968.

Guppy, M. and Withers, P. 1999. Metabolic depression in animals: physiological perspectives and biochemical generalizations. - Biological Reviews of the Cambridge Philosophical Society 74(1): 1-40.

Heckmann, L., Drossel, B., Brose, U. and Guill, C. 2012. Interactive effects of bodysize structure and adaptive foraging on food-web stability: Body size, adaptivity and food-web stability. - Ecology Letters 15(3): 243-250.

Hudson, L. N., Isaac, N. J. B. and Reuman, D. C. 2013. The relationship between 
body mass and field metabolic rate among individual birds and mammals. - Journal of Animal Ecology 82(5): 1009-1020.

Hudson, L. N. and Reuman, D. C. 2013. A cure for the plague of parameters: constraining models of complex population dynamics with allometries. - Proc. R. Soc. B 280(1770): 20131901.

Jeyasingh, P. D. 2007. Plasticity in metabolic allometry: the role of dietary stoichiometry. - Ecology Letters 10(4): 282-289.

Jones, S. E. and Lennon, J. T. 2010. Dormancy contributes to the maintenance of microbial diversity. - Proceedings of the National Academy of Sciences 107(13): 5881-5886.

Kalinkat, G., Schneider, F. D., Digel, C., Guill, C., Rall, B. C. and Brose, U. 2013. Body masses, functional responses and predator-prey stability. - Ecology Letters 16(9): $1126-1134$.

Kartascheff, B., Heckmann, L., Drossel, B. and Guill, C. 2010. Why allometric scaling enhances stability in food web models. - Theoretical Ecology 3(3): 195-208.

Kondoh, M. 2003. Foraging adaptation and the relationship between food-web complexity and stability. - Science 299(5611): 1388-1391.

- 2010. Linking learning adaptation to trophic interactions: a brain size-based approach. - Functional Ecology 24(1): 35-43.

Křivan, V. and Diehl, S. 2005. Adaptive omnivory and species coexistence in tri-trophic food webs. - Theoretical Population Biology 67(2): 85-99.

Kuwamura, M., Nakazawa, T. and Ogawa, T. 2009. A minimum model of prey-predator system with dormancy of predators and the paradox of enrichment. - Journal of Mathematical Biology 58(3): 459-479. 
Lang, B., Rall, B. C. and Brose, U. 2012. Warming effects on consumption and intraspecific interference competition depend on predator metabolism. - Journal of Animal Ecology 81(3): 516-523.

Loreau, M. and de Mazancourt, C. 2013. Biodiversity and ecosystem stability: a synthesis of underlying mechanisms. - Ecology Letters 16: 106-115.

MacArthur, R. 1955. Fluctuations of animal populations and a measure of community stability. - Ecology 36(3): 533.

Magozzi, S. and Calosi, P. 2015. Integrating metabolic performance, thermal tolerance, and plasticity enables for more accurate predictions on species vulnerability to acute and chronic effects of global warming. - Global Change Biology 21(1): 181-194.

Makarieva, A. M., Gorshkov, V. G. and Li, B.-L. 2005. Energetics of the smallest: do bacteria breathe at the same rate as whales?. - Proceedings of the Royal Society B: Biological Sciences 272(1577): 2219-2224.

Makarieva, A. M., Gorshkov, V. G., Li, B.-L., Chown, S. L., Reich, P. B. and Gavrilov, V. M. 2008. Mean mass-specific metabolic rates are strikingly similar across life's major domains: Evidence for life's metabolic optimum. - Proceedings of the National Academy of Sciences 105(44): 16994-16999.

Marshall, D. J., Dong, Y.-w., McQuaid, C. D. and Williams, G. A. 2011. Thermal adaptation in the intertidal snail Echinolittorina malaccana contradicts current theory by revealing the crucial roles of resting metabolism. - Journal of Experimental Biology 214(21): 3649-3657.

Marshall, D. J. and McQuaid, C. D. 2011. Warming reduces metabolic rate in marine 
snails: adaptation to fluctuating high temperatures challenges the metabolic theory of ecology. - Proceedings of the Royal Society B: Biological Sciences 278(1703): 281-288.

May, R. M. 1972. Will a large complex system be stable?. - Nature 238(5364): 413-414.

McCann, K. S. 2000. The diversity-stability debate. - Nature 405(6783): 228-233.

Montoya, J. M., Pimm, S. L. and Solé, R. V. 2006. Ecological networks and their fragility.Nature 442(7100): 259-264.

Mougi, A. and Nishimura, K. 2007. A resolution of the paradox of enrichment. - Journal of Theoretical Biology 248(1): 194-201.

- 2008. The paradox of enrichment in an adaptive world. - Proceedings of the Royal Society B: Biological Sciences 275(1651): 2563-2568.

Nakazawa, T., Kuwamura, M. and Yamamura, N. 2011. Implications of resting eggs of zooplankton for the paradox of enrichment. - Population Ecology 53(2): 341-350.

Norin, T., Malte, H. and Clark, T. D. 2015. Differential plasticity of metabolic rate phenotypes in a tropical fish facing environmental change. - Functional Ecology pp. $\mathrm{n} / \mathrm{a}-\mathrm{n} / \mathrm{a}$.

Otto, S. B., Rall, B. C. and Brose, U. 2007. Allometric degree distributions facilitate food-web stability. - Nature 450(7173): 1226-1229.

Pawar, S., Dell, A. I. and Savage, V. M. 2012. Dimensionality of consumer search space drives trophic interaction strengths. - Nature .

Pimm, S. L. 1984. The complexity and stability of ecosystems. - Nature 307(5949): $321-326$. 
Polis, G. A., Holt, R. D., Menge, B. A. and Winemiller, K. O. 1996. Time, space, and life history: influences on food webs. - In: Polis, G. A. and Winemiller, K. O. (eds.), Food Webs. Springer US, pp. 435-460.

Rall, B., Guill, C. and Brose, U. 2008. Food-web connectance and predator interference dampen the paradox of enrichment. - Oikos 117(2): 202-213.

Ramasubramanian, K. and Sriram, M. S. 2000. A comparative study of computation of Lyapunov spectra with different algorithms. - Physica D: Nonlinear Phenomena 139(12): 72-86. ArXiv: chao-dyn/9909029.

Rip, J. M. K. and McCann, K. S. 2011. Cross-ecosystem differences in stability and the principle of energy flux. - Ecology Letters 14(8): 733-740.

Rooney, N. and McCann, K. S. 2012. Integrating food web diversity, structure and stability. - Trends in Ecology \& Evolution 27(1): 40-46.

Rosenzweig, M. L. 1971. Paradox of enrichment: destabilization of exploitation ecosystems in ecological time. - Science (New York, N.Y.) 171(3969): 385-387.

Roy, S. and Chattopadhyay, J. 2007. The stability of ecosystems: A brief overview of the paradox of enrichment. - Journal of Biosciences 32(2): 421-428.

de Ruiter, P. C. 2005. Food web ecology: playing Jenga and beyond. - Science 309(5731): $68-71$.

Savage, V. M., Gillooly, J. F., Woodruff, W. H., West, G. B., Allen, A. P., Enquist, B. J. and Brown, J. H. 2004. The predominance of quarter-power scaling in biology.Functional Ecology 18(2): 257-282.

Schwarzmüller, F., Eisenhauer, N. and Brose, U. 2015. 'Trophic whales' as biotic buffers: 
weak interactions stabilize ecosystems against nutrient enrichment. - Journal of Animal Ecology 84(3): 680-691.

Shimada, I. and Nagashima, T. 1979. A numerical approach to ergodic problem of dissipative dynamical systems. - Progress of Theoretical Physics 61(6): 1605-1616.

Skalski, G. T. and Gilliam, J. F. 2001. Functional responses with predator interference: viable alternatives to the Holling type II model. - Ecology 82(11): 3083-3092.

Uchida, S., Drossel, B. and Brose, U. 2007. The structure of food webs with adaptive behaviour. - Ecological Modelling 206(3-4): 263-276.

Verschoor, A. M., Vos, M. and Van Der Stap, I. 2004. Inducible defences prevent strong population fluctuations in bi- and tritrophic food chains. - Ecology Letters 7(12): 11431148.

Vos, M., Kooi, B. W., DeAngelis, D. L. and Mooij, W. M. 2004. Inducible defences and the paradox of enrichment. - Oikos 105(3): 471-480.

Wang, J. and Jiang, W. 2014. Impulsive perturbations in a predator-prey model with dormancy of predators. - Applied Mathematical Modelling 38(9-10): 2533-2542.

Williams, R. J., Brose, U. and Martinez, N. D. 2007. Homage to Yodzis and Innes 1992: Scaling up feeding-based population dynamics to complex ecological networks. - In: From Energetics to Ecosystems: The Dynamics and Structure of Ecological Systems. Springer, Dordrecht, pp. 37-51. DOI: 10.1007/978-1-4020-5337-5_2.

Williams, R. J. and Martinez, N. D. 2000. Simple rules yield complex food webs. - Nature 404(6774): 180-183.

Wolf, A., Swift, J. B., Swinney, H. L. and Vastano, J. A. 1985. Determining Lyapunov exponents from a time series. - Physica D: Nonlinear Phenomena 16(3): 285-317. 
Yashchenko, V., Fossen, E. I., Kielland, Ø. N. and Einum, S. 2016. Negative relationships between population density and metabolic rates are not general. - Journal of Animal Ecology 85(4): 1070-1077.

Yodzis, P. and Innes, S. 1992. Body size and consumer-resource dynamics. - The American Naturalist 139(6): 1151. 
Table 1: Parameters and variables used in the model

\begin{tabular}{ccl}
\hline Variable & Value & Description \\
\hline$B_{i}$ & kg.m ${ }^{-2}$ & biomass density of species $i$ \\
$r_{i}$ & dimensionless & scaled mass specific maximum growth rate of species $i$ \\
$x_{i}$ & dimensionless & scaled mass specific metabolic rate of species $i$ \\
$y_{i}$ & 8 & scaled mass specific maximum consumption rate \\
$e_{j i}$ & 0.45 & assimilation efficiency of species $i$ by species $j$ (herbivores) \\
& 0.85 & assimilation efficiency of species $i$ by species $j$ (carnivores) \\
$G_{i}$ & dimensionless & density dependent growth rate of species $i$ \\
$F_{i j}$ & dimensionless & functional response of species $i$ feeding on species $j$ \\
$B_{0}$ & $0.5 \mathrm{~kg} \cdot \mathrm{m}^{-2}$ & half saturation density for consumer functional response \\
$c$ & $0.5 \mathrm{~m}^{2} \cdot \mathrm{kg}^{-1}$ & predator interference \\
$\omega_{i j}$ & $1 / \mathrm{nbr} \mathrm{prey}$ & predator $i$ preference for species $j$ \\
$a_{x} / a_{r}$ & 0.138 & metabolic rate allometric constant (primary producers) \\
& 0.314 & metabolic rate allometric constant (invertebrates consumers) \\
$X$ & dimensionless & metabolic adjustment coefficient \\
$K$ & kg.m ${ }^{-2}$ & carrying capacity of primary producers
\end{tabular}

Note: All these parameters come from Brose et al. (2006). 
(A)

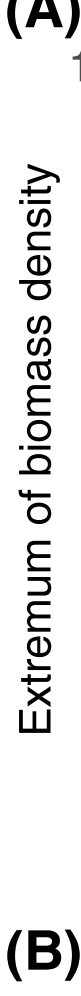

(B)

\subsection{0}

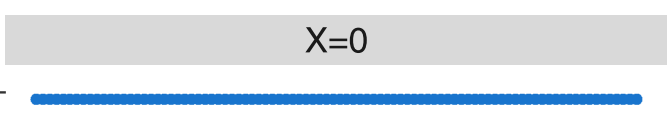

$X=0$

- Carnivore

- Herbivore

- Primary producer

7.5

5.0

2.5.
0.0

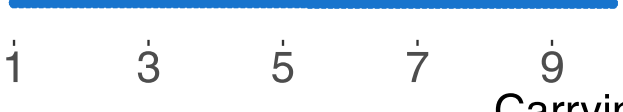

$X=2$

0.8

$0.6-$

$0.4-$

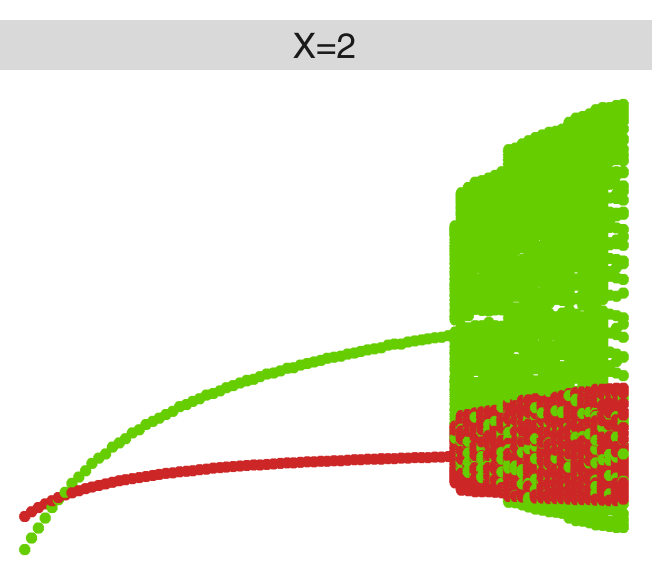

0.0

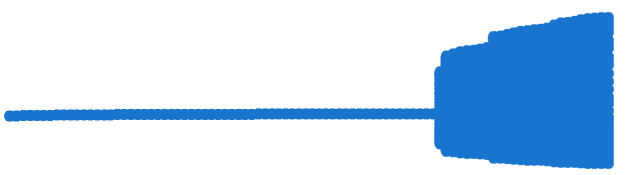

1

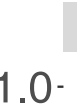

$X=2$

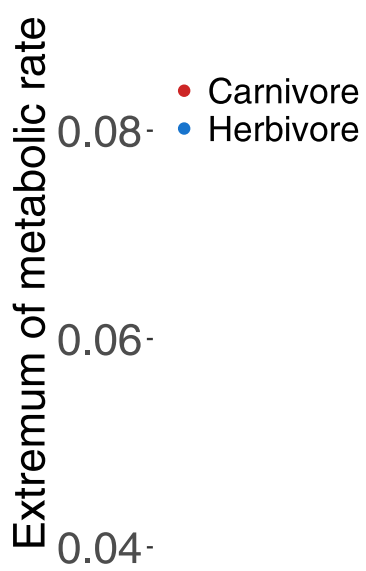

0.8

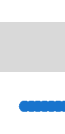

\section{3}

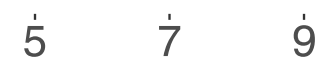

9

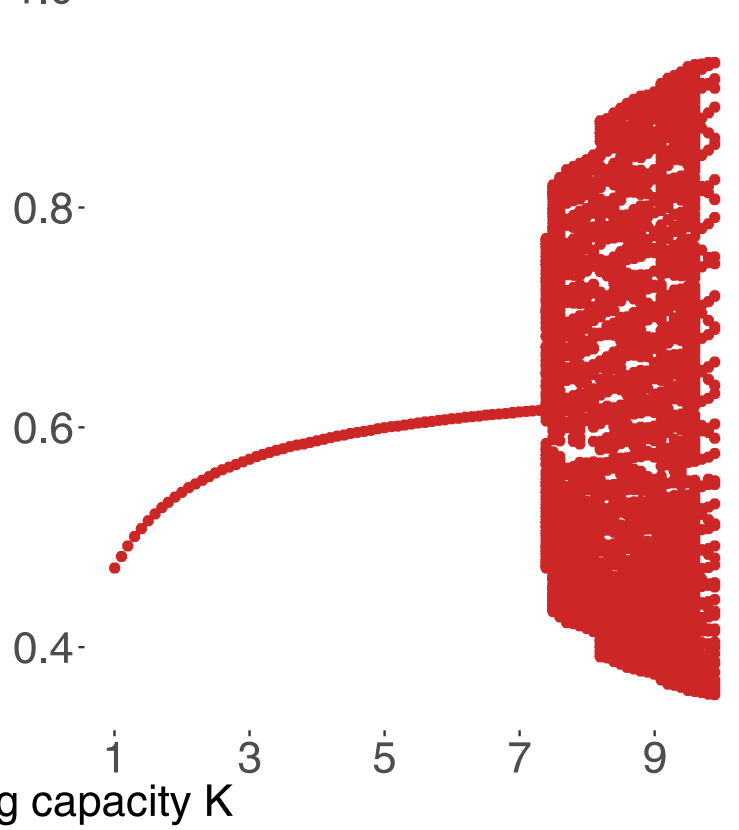

Figure 1: Bifurcation diagrams of the tri-trophic food-chain containing a primary producers (green), a herbivores (blue) and a carnivores (red). The bifurcation is performed along gradients in the carrying capacity $K$ for $\mathbf{A}$ ) biomass density and $\mathbf{B}$ ) metabolic rate for a metabolic adjustment coefficient $X=0$ or $X=2$. 

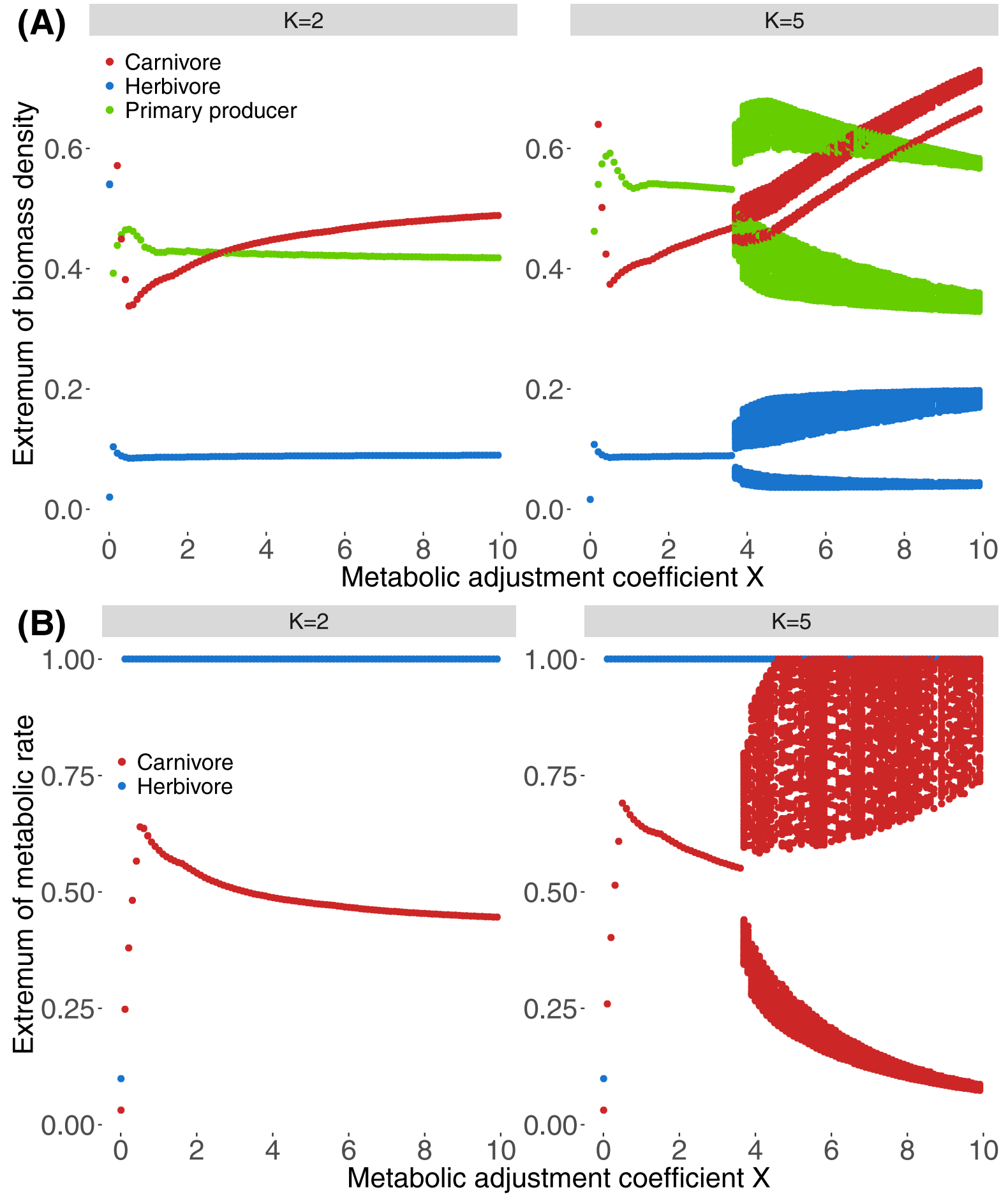

Figure 2: Bifurcation diagrams of the tri-trophic food-chain containing a primary producers (green), a herbivores (blue) and a carnivores (red). The bifurcation is performed along gradients in the metabolic adjustment coefficient $X$ for A) biomass density and B) metabolic rate for a carrying capacity $K=1$ or $K=2$. 

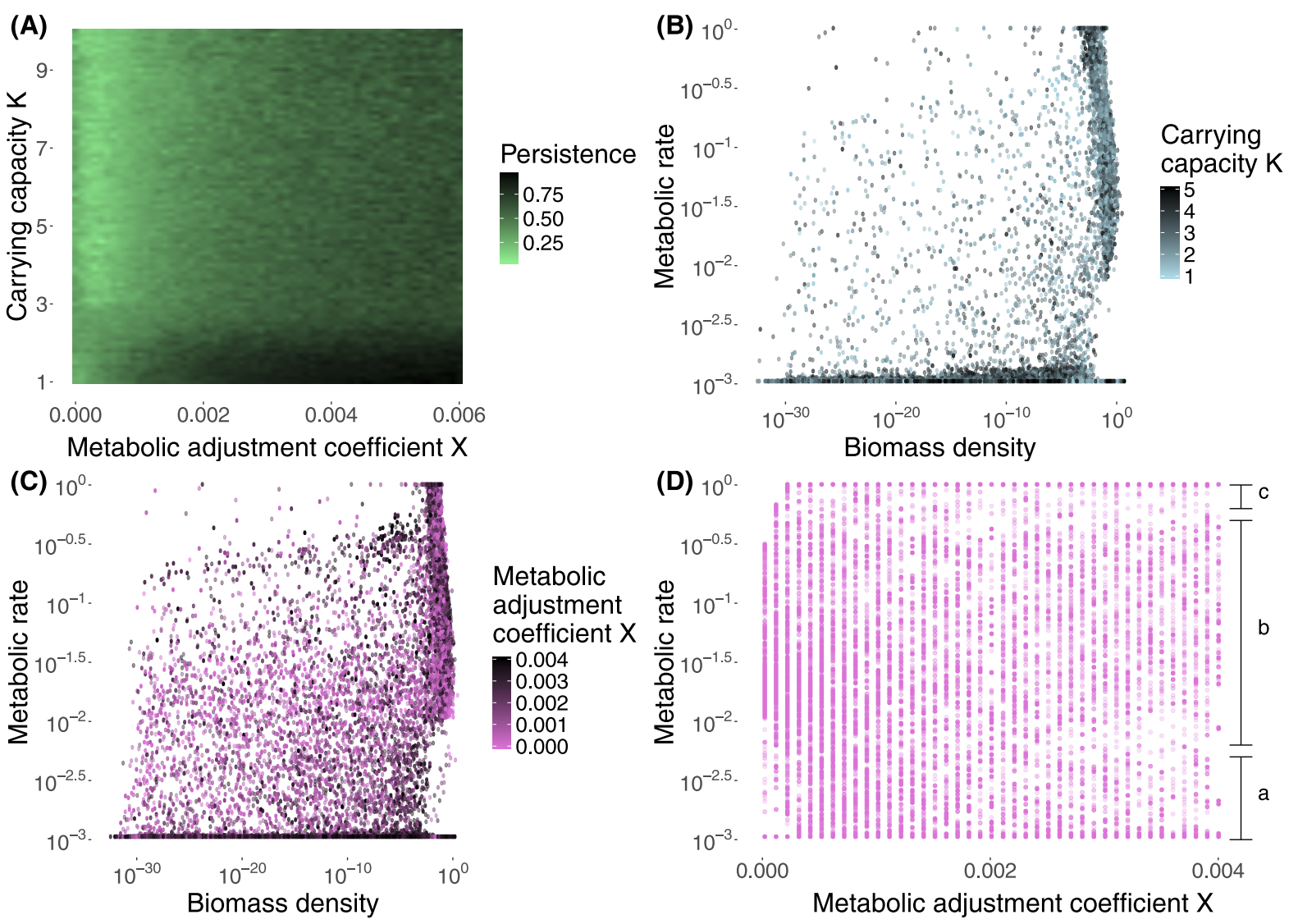

Figure 3: Effects of metabolic adjustment on complex food webs. A) Persistence of species for different values of metabolic adjustment coefficient $X$ and carrying capacity $K$. Each square represent the average persistence for 100 replicates. B) Metabolic rate versus biomass density along gradient in carrying capacity $K(X=0.004)$. C)Metabolic rate versus biomass density along a metabolic adjustment coefficient gradient $(K=1.5)$. Each point represents one species and 100 food webs are tested for each combination of $K$ and $X$. D) Distribution of the average metabolic rate of each species along a metabolic adjustment coefficient gradient $(K=1.5)$. The domains a, b and c represent respectively species with minimum or low metabolic rate, species with intermediate metabolic rate and species with maximum metabolic rate. 